\title{
Monte Carlo simulation with time step quantification in terms of Langevin dynamics
}

\author{
U. Nowak ${ }^{1}$, R. W. Chantrell ${ }^{2}$, and E. C. Kennedy ${ }^{3}$ \\ ${ }^{1}$ Theoretische Tieftemperaturphysik, Gerhard-Mercator-Universität-Duisburg, 47048 Duisburg, Germany \\ 2 SEES, University of Wales Bangor, Dean Street, Bangor, LL57 1UT, UK \\ ${ }^{3}$ Dept. of Appl. Maths and Theo. Phys., The Queen's University of Belfast, Belfast, BTr 1NN, Northern Ireland
}

\begin{abstract}
For the description of thermally activated dynamics in systems of classical magnetic moments numerical methods are desirable. We consider a simple model for isolated magnetic particles in a uniform field with an oblique angle to the easy axis of the particles. For this model, a comparison of the Monte Carlo method with Langevin dynamics yields new insight in the interpretation of the Monte Carlo process, leading to the implementation of a new algorithm where the Monte Carlo step is time-quantified. The numeric results for the characteristic time of the magnetisation reversal are in excellent agreement with asymptotic solutions which itself are in agreement with the exact numerical results obtained from the Fokker-Planck equation for the Néel-Brown model.
\end{abstract}

Studies of spin dynamics in particulate systems are currently of significant interest, as model systems for understanding the thermodynamics of the reversal process. Brown [1] developed a theoretical formalism for thermally activated magnetisation reversal based on the FokkerPlanck (FP) equation which led to a high energy barrier asymptotic formula in the axially symmetric case of a particle with easy (uniaxial) anisotropy axis collinear with the applied magnetic field. Since then extensive calculations [2 [6] have been carried out in which improved approximations were found for the axially symmetric case. Coffey and co-workers [4] 6] also derived formulae for the non-axially symmetric case, investigating also the different regimes imposed by the damping parameter $\alpha$ of the Landau-Lifshitz-Gilbert (LLG) equation. This work represents an important basis for the understanding of dynamic processes in single-domain particles. New experimental techniques which allow for an investigation of nanometer-sized, isolated, magnetic particles confirmed this theoretical approach to thermal activation [7].

Unfortunately, the extension of this work to the important case of strongly coupled spin systems such as are found in micromagnetic calculations of magnetisation reversal is non-trivial, and realistic calculations in systems with many degrees of freedom would appear to be impossible except by computational approaches. These are currently of two types: (i) calculations involving the direct simulation of the stochastic (Langevin) equation of the problem, in this case the LLG equation supplemented by a random force representing the thermal perturbations. This is referred to as the Langevin Dynamics (LD) formalism [8.9, and (ii) Monte Carlo (MC) simulations 10] with a continuously variable (Heisenberg like) Hamiltonian [11,12]. The LD approach, although having a firm physical basis is limited to timescales of the order of a few ns for strongly coupled systems. The MC approach is capable of studying longer timescales involving rever- sal over large energy barriers, but has the severe problem of having no physical time associated with each step, resulting in unquantified dynamic behavior.

Physically, the dynamic behavior of interacting spin systems is a topic of considerable current interest, much of this interest being driven by the need to understand spin electronic devices such as MRAM. The possibility of truly dynamic models of strongly coupled systems would seem to be an important factor in the development of a fundamental physical understanding. This requires dynamic studies over the whole time range from ns and sub - ns to the so-called 'slow dynamic' behavior arising from thermally excited decay of metastable states over timescales from 10-100s and upwards. It is inconceivable that the LD technique can be used over the whole timescale and therefore a truly time quantified MC technique is necessary in order to allow calculations over the longer timescales of physical interest. Here we propose a technique for the quantification of the MC timestep and give a supporting argument developed from the fluctuation dissipation theorem. This argument results in a theoretical expression for the timestep in terms of the size of MC move, and also gives the validity criterion that the MC timestep is much longer than the precession time. Comparison with an analytical formula for relaxation in the intermediate to high damping limit is used to verify the theoretically predicted relationship relating the timestep to the size of MC move. This represents an important first step in the process of deriving a theoretical formalism for time quantified $\mathrm{MC}$ calculations of strongly interacting spin systems.

We consider an ensemble of isolated single-domain particles where each particle is represented by a magnetic moment with energy

$$
E(\underline{S})=-d V S_{z}^{2}-\mu_{s} \underline{B} \cdot \underline{S},
$$

where $\underline{S}=\underline{\mu} / \mu_{s}$ is the magnetic moment of unit length, $\underline{B}=B_{x} \underline{\hat{x}}+B_{z} \underline{\underline{z}}$ represents a magnetic field under an arbitrary angle $\psi$ to the easy axis of the system, $d$ is the 
uniaxial anisotropy energy density and $V$ the volume of the particle. Throughout the article we use the material parameters $V=8 \times 10^{-24} \mathrm{~m}^{3}, d=4.2 \times 10^{5} \mathrm{~J} / \mathrm{m}^{3}$, magnetic moment $\mu_{s}=1.12 \times 10^{-17} \mathrm{~J} / \mathrm{T}$.

The LLG equation of motion with LD [1] is

$$
\underline{\dot{S}}=-\frac{\gamma}{\left(1+\alpha^{2}\right) \mu_{s}} \underline{S} \times(\underline{H}(t)+\alpha \underline{S} \times \underline{H}(t)),
$$

where $\gamma=1.76 \cdot 10^{11}(T s)^{-1}$ is the gyromagnetic ratio, $\underline{H}(t)=\underline{\zeta}(t)-\frac{\partial E}{\partial \underline{S}}$, and $\zeta$ is the thermal noise with $\left\langle\zeta_{i}(t)\right\rangle=0$ and $\left\langle\zeta_{i}(t) \zeta_{j}\left(t^{\prime}\right)\right\rangle=\delta_{i j} \delta\left(t-t^{\prime}\right) 2 \alpha k_{B} T \mu_{s} / \gamma . i$ and $j$ denote the cartesian components.

The equation above is solved numerically using the Heun method [9]. Also, it is possible to obtain analytically asymptotic solutions for the escape rate which have been extensively compared with the exact numerical solutions from the corresponding matrix form of the FP equation for a wide range of parameters and non-axially symmetric potentials 46 .

Both of our simulations, MC as well as LD, start with the magnetic moments in $z$-direction. The magnetic field has a negative $z$-component so that the magnetization will reverse after some time. The time that is needed for the $z$-component of the magnetization to change its sign averaged over a large number of runs $(N=1000)$ is the characteristic time $\tau$ which corresponds to the inverse of the escape rate following from exact numerical solutions of the corresponding FP equation.

For the MC simulations we use a heat-bath algorithm. The trial step of our MC algorithm is a random movement of the magnetic moment within a cone with a given size. In order to achieve this efficiently we construct a random vector with constant probability distribution within a sphere of radius $R$. This random vector is added to the initial moment and subsequently the resulting vector is normalized.

The size of the cone $R$ of our algorithm influences the time scale the method simulates. We investigate the influence of $R$ on our MC algorithm by varying $R$ and calculating $\tau$. As usual in a MC procedure the time is measured in Monte Carlo steps (MCS). For our calculation we use a field of $|\underline{B}|=0.2 \mathrm{~T}$ and an angle of $\psi=27^{\circ}$ to the easy axis. The resulting energy barrier is $\Delta E=8.2 \times 10^{-19} \mathrm{~J}$, the temperature we chose for Fig. 1 is $\Delta E / k_{B} T=3.3$. As Fig. 目 demonstrates, it is $\tau \sim R^{-2}$. This dependence can be understood by considering the moments as performing a random walk where $R$ is proportional to the mean step width. Having understood that the MC time can be set by choosing an appropriate size of the step width we search for a relation for $R$ such that one MCS corresponds to a real-time interval, in the sense of LD.

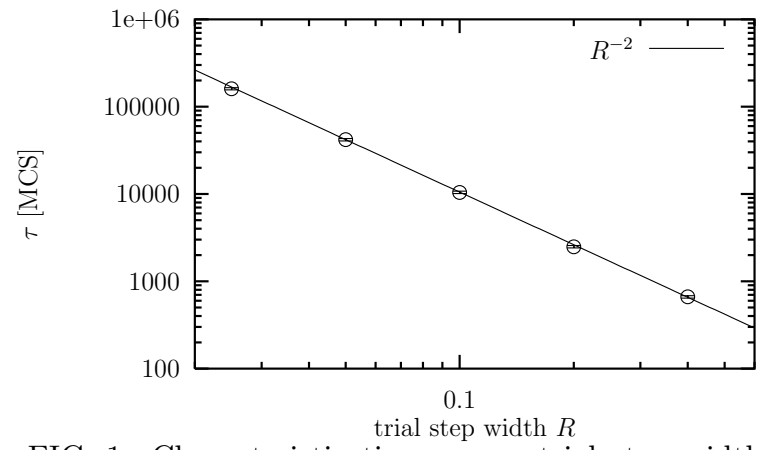

FIG. 1. Characteristic time versus trial step width for a MC simulation. The solid line is fitted, yielding $\tau \sim R^{-2}$.

MC methods calculate trajectories in phase space following a master equation which describes the coupling of a system to the heat bath. Hence, only the irreversible part of the dynamics of the system is considered [13] there is no precession of the moments since no equation of motion is solved during the simulation. Nevertheless, in the following we will argue that the exact knowledge of the movement of the single moments is not necessary in order to describe the effects of thermal activation in an ensemble of systems under the following conditions: (i) the relevant time scales are larger than the precession time $t_{p}$ of the moments, (ii) we consider the high damping limit of the LLG equation where the energy dissipation during one cycle of the precession is considerably large so that the system relaxes (to the local energy minimum) on the same time scale $t_{r} \approx t_{p}$.

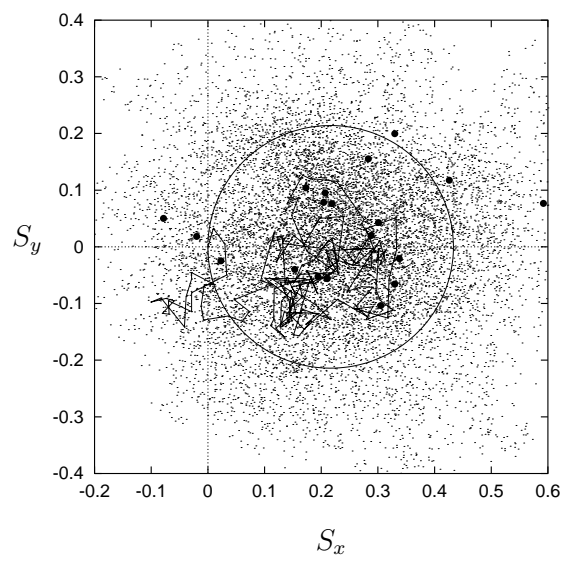

FIG. 2. Configuration in phase space $\left(S_{x}, S_{y}\right)$ of an ensemble of 20 particles following from a LD simulation for $\alpha=1$ : see text for details.

In Fig. 2 we present the time evolution of our system in phase space, $\left(S_{x}, S_{y}\right)$, following from a simulation of the LLG equation for high damping, $\alpha=1$. We use $\Delta E / k_{B} T=8.2$, a rather low temperature so that the characteristic time $\tau$ for the escape from the local energy minimum is of the order of $10^{-6} \mathrm{~s}$ (see also Fig. 3). 
The spin-precession time is $t_{p}=9 \times 10^{-11} \mathrm{~s}$ here. The simulation starts close to the local energy minimum with $S_{x}=S_{y}=0, S_{z}=1$ and the solid line shows the trajectory of one moment over a time interval of $\Delta t=t_{p}$. The 20 points are the positions of an ensemble of 20 moments after the same time. As one can see, the moments show no significant precession (the precession of an undisturbed moment, i. e. without relaxation and fluctuations is indicated by the circle around the energy minimum at $S_{y}=0, S_{x} \approx 0.22$ ). The small dots represent 1000 states of the ensemble for $t<6 \times t_{p}$. Altogether, Fig. 2 demonstrates that in the high damping case already after time periods of only a few $t_{p}$ the moments are uncorrelated and the ensemble reaches a local equilibrium configuration (remember that the time scale to leave the local equilibrium is much larger here so that Fig. 2 shows only the local short-time equilibration, not the escape from the local energy minimum).

We will show that this high-damping scenario can also be simulated by a MC simulation and we will now derive a relation for $R$ in order to quantify the MC time step. The intention is to compare the fluctuations which are established in the MC technique within one MCS with the fluctuations within a given time scale associated with the linearized LLG equation. Close to a local energy minimum one can write the energy, given that first order terms vanish as

$$
E \approx E_{0}+\frac{1}{2} \sum_{i, j} A_{i j} S_{i} S_{j},
$$

where the $S_{i}$ are the variables representing small deviations from equilibrium. In our system, for $B_{x}=0$ we find equilibrium along the $z$ axis, leading to variables $S_{x}$ and $S_{y}$. The energy increase $\Delta E$ associated with fluctuation in $S_{x}$ and $S_{y}$ is $\Delta E \approx \frac{1}{2}\left(A_{x x} S_{x}^{2}+A_{y y} S_{y}^{2}\right)$, with $A_{x x}=A_{y y}=2 d V+\mu_{s} B_{z}$. Rewriting the LLG equation in the linearized form, $\dot{S}_{x}=L_{x x} S_{x}+L_{x y} S_{y}$, $\dot{S}_{y}=L_{y x} S_{x}+L_{y y} S_{y}$, it has been shown [14] that the correlation function takes the form

$$
\left\langle S_{i}(t) S_{j}\left(t^{\prime}\right)\right\rangle=\mu_{i j} \delta_{i, j} \delta\left(t-t^{\prime}\right) .
$$

Dirac's $\delta$ function is here an approximation for exponentially decaying correlations on time scales $t-t^{\prime}$ that are much larger than the time scale of the exponential decay $t_{r}$. The covarianz matrix $\mu_{i j}$ can be calculated from the system matrices $\mathbf{A}$ and $\mathbf{L}$ as [14] $\mu_{i j}=$ $-k_{B} T\left(L_{i k} A_{k j}^{-1}+L_{j k} A_{k i}^{-1}\right)$. For our problem a short calculation yields $\mu_{x x}=\mu_{y y}=2 k_{B} T \frac{\alpha \gamma}{\left(1+\alpha^{2}\right) \mu_{s}}$. Integrating the fluctuating magnetisation $S_{x}(t)$ over a finite time interval $\Delta t$, Eq. 国 takes the form

$$
\left\langle\bar{S}_{x}^{2}\right\rangle=\mu_{x x} \Delta t=2 k_{B} T \frac{\alpha \gamma}{\left(1+\alpha^{2}\right) \mu_{s}} \Delta t,
$$

representing the fluctuations of $S_{x}$ averaged over a time interval $\Delta t$.
Next, we calculate the fluctuations $\left\langle S_{x}^{2}\right\rangle$ during one MCS of a MC simulation. This is possible if we assume that all magnetic moments are initially in their equilibrium position. For our MC algorithm described above the probability distribution for trial steps with step width $r=\sqrt{S_{x}^{2}+S_{y}^{2}}$ is $p_{\mathrm{t}}=3 \sqrt{R^{2}-r^{2}} /\left(2 \pi R^{3}\right)$. The acceptance probability within a heat bath algorithm is $p_{\mathrm{a}}(r)=1 /\left(1+\exp \left(\Delta E\left(r^{2}\right) / k_{B} T\right)\right)$, where $\Delta E\left(r^{2}\right)$ can be taken from Eq. 3. Hence, for the fluctuations within one MC step it is:

$$
\left\langle S_{x}^{2}\right\rangle=2 \pi \int_{0}^{R} r \mathrm{~d} r \frac{r^{2}}{2} p_{\mathrm{t}}(r) p_{\mathrm{a}}(r)=\frac{R^{2}}{10}+\mathcal{O}\left(R^{4}\right)
$$

where the last line is an expansion for small $R$. By equalizing the fluctuations within corresponding time intervals we find the relation

$$
R^{2}=\frac{20 k_{B} T \alpha \gamma}{\left(1+\alpha^{2}\right) \mu_{s}} \Delta t
$$

Note, from our derivation above it follows that one time step $\Delta t$ must be larger than the intrinsic time scale $t_{r}$ of the relaxation. This means - as already mentioned above - that the Monte Carlo method can only work on time scales that are much larger than any microscopic time scale of a precession or relaxation (to local equilibrium) of the moment.

In principle, equation 7 gives the possibility to choose the trial step for a MC simulation in such a way that $1 \mathrm{MCS}$ corresponds to a real time interval, say $\Delta t=$ $10^{-12} \mathrm{~s}$. However, there are of course restrictions for possible values of $R$, like $R<1$. Also, $R$ should not be too small since then a Monte Carlo algorithm is inefficient. Therefore, either one has to choose such a value for $\Delta t$ so that $R$ takes on reasonable values (these will usually be of the order of $10^{-12} \mathrm{~s}$ ) or one uses a reasonable constant value for $R$, say 0.1 , and uses Eq. 7 to calculate $\Delta t$ as the real time interval corresponding to 1MCS. In the following we use the first method since it turns out to be very efficient to change $R$ with temperature. However, we confirmed that the other method yields the same results.

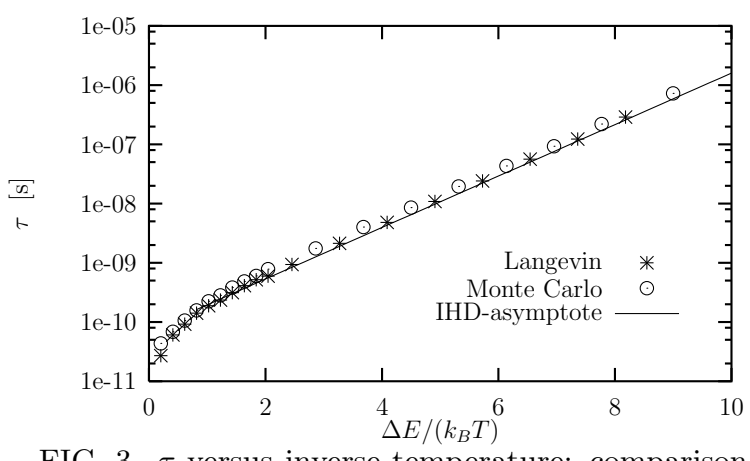

FIG. 3. $\tau$ versus inverse temperature: comparison of IHD asymptote, LD simulation, and MC simulation. 
To test the validity of our considerations we performed MC simulations with an algorithm using a trial step according to Eq. 7 with $\Delta t \approx 6 \times 10^{-12}$ s (the inverse value of $\gamma$, in other words the time in the LLG equation is rescaled by $\gamma$ ). For Fig. 3 we set $\alpha=1$ and compare the data for $\tau(T)$ following from our MC simulation with results from $\mathrm{LD}$ simulations and with the intermediate to high damping (IHD) asymptote [5, 6], namely

$$
\tau=\frac{2 \pi \omega_{0}}{\Omega_{0} \omega_{2}} e^{\beta\left(V_{0}-V_{2}\right)}=\frac{2 \pi \omega_{0}}{\Omega_{0} \omega_{2}} e^{\Delta E / k_{B} T},
$$

where $\omega_{0}$ and $\Omega_{0}$ are the saddle and damped saddle angular frequencies which have been defined in Eqs. (21) and (22) of Ref. [6] explicitly. $\omega_{2}$ is the well angular frequency for the deeper of the two potential wells and is defined in Eq. (20) of Ref. [6]. All have been defined in terms of the coefficients of the truncated Taylor series representation of the energy equation described in detail in section V of Ref. [4], (particularly Eqs. 59-64). For the purpose of comparison with MC and LD simulations, we consider one escape path only, $e^{\beta\left(V_{0}-V_{2}\right)}$, where $\beta=V / k_{B} T$ and $V_{0}-V_{2}$ is the energy described by Eq. (62) of Ref. [4. For our purposes, $\beta\left(V_{0}-V_{2}\right)$ may be represented by $\Delta E / k_{B} T$. The validity condition for the IHD formula is $\alpha \Delta E / k_{B} T \gg 1$ where $\Delta E / k_{B} T>1$ which have been satisfied in all cases represented here.

From Fig. 3 it is clear that the LD data agree with the asymptote above. For higher temperatures the asymptote is no longer appropriate. Here, the numerical data for $\tau$ tend to zero for $T \rightarrow \infty$ as one expects. The MC data deviate slightly and are roughly 10\% larger. However, considering the fact that to the best of our knowledge this is the first comparison of a "real-time MC simulation" with LD simulations and asymptotic formulae, the agreement is remarkable - especially taking into account the simple form of Eq.7 underlying our algorithm and also that there is no adjusted parameter in all our calculations and formulae.

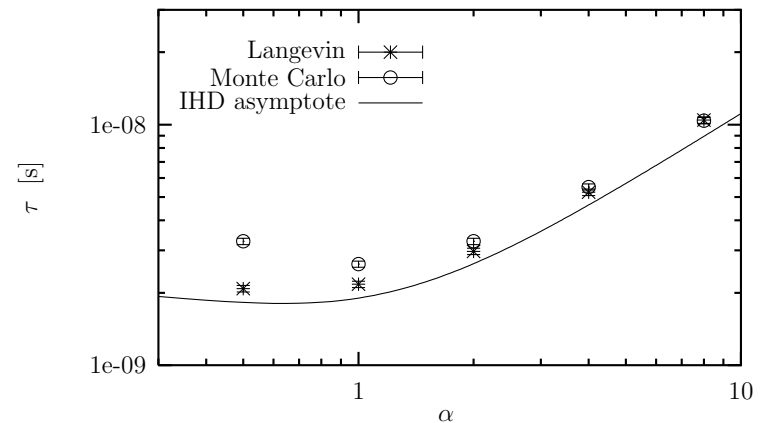

FIG. 4. $\tau$ versus damping constant: comparison of IHD asymptote, LD simulation, and MC simulation.

Since we expect that our MC procedure leads to a high damping limit we also tested the $\alpha$-dependence of $\tau$. Fig. 1 shows the corresponding data for the same parameter values as before and $\Delta E / T=3.3$. The figure shows that the $\mathrm{MC}$ data converge to the IHD formula and to the data from LD simulation for large $\alpha$. Even the small $10 \%$ deviation of the MC data mentioned before (Fig. 3) vanishes in the limit of larger $\alpha$.

To summarize, we discussed the conditions under which a comparison of LD with a MC process appears to be possible. Considering a simple system of isolated single-domain particles we derived an equation for the trial step width of the MC process so that one step of the MC algorithm can be related to a certain time interval. Testing this algorithm we found excellent agreement with data from LD simulation as well as with intermediate to high damping asymptotes for the characteristic times of the magnetisation reversal. Even, though our algorithm was derived only for the special system which we consider here, we belive that the arguments we brought forward might the fundament even for the MC simulation of more complicated systems, especially systems consisting of interacting magnetic moments.

\section{ACKNOWLEDGMENTS}

We would like to thank W. T. Coffey and K. D. Usadel for helpful discussions. E. C. Kennedy thanks EPSRC for financial support (GR/L06225). R. W. Chantrell thanks EPSRC for financial support (ref GR/M24011). This work was done within the framework of the COST action P3 working group 4.

** e-mail address: uli@thp.uni-duisburg.de

[1] W. F. Brown, Phys. Rev. 130, 1677 (1963).

[2] I. Klik and L. Gunther, J. Stat. Phys. 60, 473 (1990).

[3] H. B. Braun, Phys. Rev. Lett. 71, 3557 (1993) and J. Appl. Phys. 85, 6127 (1999).

[4] W. T. Coffey, D. S. F. Crothers, J. L. Dormann, L. J. Geoghegan, E. C. Kennedy, Phys. Rev. B 58, 3249, (1998).

[5] W. T. Coffey, D. S. F. Crothers, J. L. Dormann, Yu. P. Kalmikov, E. C. Kennedy, W. Wernsdorfer, Phys. Rev. Lett. 80, 5655 (1998).

[6] W. T. Coffey, D. S. F. Crothers, J. L. Dormann, L. J. Geoghegan, E. C. Kennedy, W. Wernsdorfer, J, Phys.: Condens. Matter 10, 9093 (1998).

[7] W. Wernsdorfer, E. Bonet Orozco, K. Hasselbach, A. Benoit, B. Barbara, N. Demoncy, A. Loiseau, H. Pascard, and D. Mailly, Phys. Rev. Lett. 78, 1791 (1997).

[8] R. W Chantrell, J. D. Hannay, M. Wongsam, T. Schrefl and H.- J, Richter, IEEE Trans. Mag., 34, 1839 (1998).

[9] J. L. García-Palacios and F. J. Lázaro, Phys. Rev. B. 58, 14937 (1998).

[10] K. Binder and D. W. Heermann, Monte Carlo Simulation in Statistical Physics (Springer-Verlag, Berlin, 1997).

[11] J.-M. Gonzalez, R. Ramirez, R. Smirnov-Rueda and J. Gonzalez, Phys. Rev. B 5216034 (1995). 
[12] D. Hinzke and U. Nowak, Phys. Rev. B 58, 265 (1998)

[13] F. Reif, Fundamentals of statistical and thermal physics (McGraw-Hill Book Company, New York 1965), p. 548.
[14] A. Lyberatos, D. V. Berkov, R. W. Chantrell, J. Phys.: Condens. Matter 5, 8911 (1993). 